\title{
Stochastic growth dynamics and composite defects in quenched immiscible binary condensates
}

\author{
I.-K. Liu, ${ }^{1,2}$ R. W. Pattinson, ${ }^{2}$ T. P. Billam, ${ }^{3}$ S. A. Gardiner, ${ }^{3}$ S. L. Cornish,${ }^{3}$ \\ T.-M. Huang, ${ }^{4}$ W.-W. Lin, ${ }^{5}$ S.-C. Gou, ${ }^{1}$ N. G. Parker, ${ }^{2}$ and N. P. Proukakis ${ }^{2, *}$ \\ ${ }^{1}$ Department of Physics, National Changhua University of Education, Changhua 50058, Taiwan \\ ${ }^{2}$ Joint Quantum Centre (JQC) Durham-Newcastle, School of Mathematics and Statistics, Newcastle University, \\ Newcastle upon Tyne, NE1 7RU, United Kingdom \\ ${ }^{3}$ Joint Quantum Centre (JQC) Durham-Newcastle, Department of Physics, Durham University, Durham, DH1 3LE, United Kingdom \\ ${ }^{4}$ Department of Mathematics, National Taiwan Normal University, Taipei 11677, Taiwan \\ ${ }^{5}$ Department of Applied Mathematics and Shing-Tung Yau Center, National Chiao Tung University, Hsinchu 30010, Taiwan \\ (Received 5 August 2014; revised manuscript received 23 May 2015; published 18 February 2016)
}

\begin{abstract}
We study the sensitivity of coupled condensate formation dynamics on the history of initial stochastic domain formation in the context of instantaneously quenched elongated harmonically trapped immiscible two-component atomic Bose gases. The spontaneous generation of defects in the fastest condensing component, and subsequent coarse-graining dynamics, can lead to a deep oscillating microtrap into which the other component condenses, thereby establishing a long-lived composite defect in the form of a dark-bright solitary wave. We numerically map out diverse key aspects of these competing growth dynamics, focusing on the role of shot-to-shot fluctuations and global parameter changes (initial state choices, quench parameters, and condensate growth rates), with our findings also qualitatively confirmed by realistic finite-duration quenches. We conclude that phase-separated structures observable on experimental time scales are likely to be metastable states whose form is influenced by the stability and dynamics of the spontaneously emerging dark-bright solitary wave.
\end{abstract}

DOI: 10.1103/PhysRevA.93.023628

\section{INTRODUCTION}

Pattern formation and the presence of coexisting phases in spatially separated domains are an emergent feature of diverse dynamical systems throughout physics [1], chemistry [2], and biology [3]. Ultracold gases offer a highly controllable theoretical and experimental test bed for studying these phenomena, particularly in the context of condensate formation dynamics [4-6], a subject of sustained interest and significance in nonequilibrium physics, both in the ultracold gas context [7-20] and beyond [21-24]. Previous works have highlighted the generic importance of the Kibble-Zurek (KZ) mechanism in defect formation during phase transitions [25,26], connecting ultracold gases with a range of phenomena from highenergy and condensed matter physics [27-29], with composite defects [28] emerging in the context of multicomponent fields by condensation in the defect core [30].

Experiments with single-component atomic Bose-Einstein condensates (BECs) have revealed the spontaneous formation of defects in the form of vortices [31], dark solitonic vortices [32,33], and persistent currents [34] during the BEC phase transition, providing quantitative confirmation of KZ scaling [19,35-38]. In multicomponent BECs (e.g. ${ }^{87} \mathrm{Rb}-{ }^{41} \mathrm{~K}[39],{ }^{87} \mathrm{Rb}-{ }^{85} \mathrm{Rb}$ [40], and $\left.{ }^{87} \mathrm{Rb}-{ }^{133} \mathrm{Cs}[41,42]\right)$, studies have focused on the formation of two same-species BECs in a tunnel-coupled geometry [43], and quantum phase transitions in binary [44-46] and spinor Bose gases

\footnotetext{
*nikolaos.proukakis@ncl.ac.uk

Published by the American Physical Society under the terms of the Creative Commons Attribution 3.0 License. Further distribution of this work must maintain attribution to the author(s) and the published article's title, journal citation, and DOI.
}

[38,47-52]. In many scenarios, however, potentially nonuniversal postquench defect dynamics strongly influence the system behavior. Work on homogeneous spinor Bose gases $[49,50]$ has identified two distinct time scales in the process of domain formation: a short time scale for initial domain formation via the $\mathrm{KZ}$ mechanism, and a long "memory" time scale for nonlinear coarse-graining dynamics to erase traces of the initial domains. Related work indicates that characteristic memory time scales may exceed experimental system lifetimes [51,52].

In this paper, we discuss the competing growth dynamics of instantaneously quenched immiscible two-component BECs,

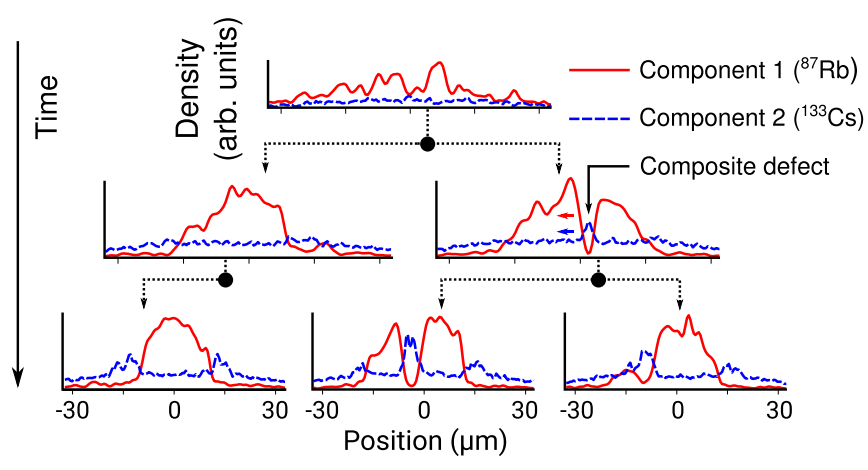

FIG. 1. Memory of stochastic defect dynamics in an immiscible two-component condensate (schematic, compiled from simulation data). On short postquench time scales, the fastest condensing component (here ${ }^{87} \mathrm{Rb}$ ) contains multiple spontaneously generated defects (top). The ensuing stochastic dynamics generically lead to either destruction of defects or survival of a single composite defect in which the second component (here ${ }^{133} \mathrm{Cs}$ ) preferentially condenses, forming a dark-bright solitary wave (middle row). The long-lived metastable states (bottom row) retain a memory of the prior stochastic dynamics. 

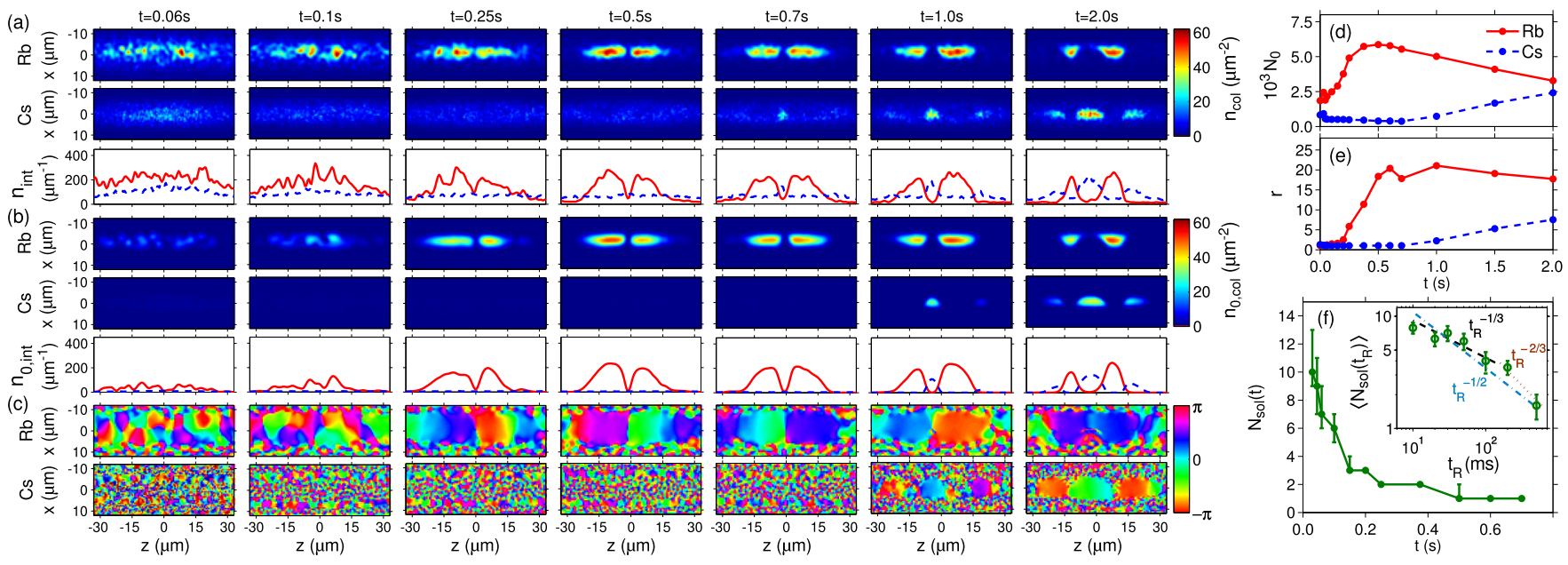

FIG. 2. Typical numerical evolution of a quenched two-component system. (a)-(b): postquench evolution, up to $2 \mathrm{~s}$, of $2 \mathrm{D}$ column densities $\left(n_{\text {col }}, n_{0, \text { col }}\right)$ and 1D integrated density profiles $\left(n_{\text {int }}, n_{0, \text { int }}\right)$ for c-field and PO (Penrose-Onsager) condensate respectively. (c): corresponding condensate phase. (d)-(e): evolution of (respectively) condensate number, $N_{0}$, and occupation ratio $r$ between condensate mode and the next-most-highly occupied mode. (f): single-run evolution of the number of spontaneously generated dark solitons, $N_{\text {sol }}(\mathrm{t})$ (obtained, to within the estimated uncertainty shown, by examining density minima and phase jumps, similar to the procedure of [36]). In all simulations in this work, both components are confined in harmonic traps with frequencies $\left(\omega_{z}^{\mathrm{Rb}}, \omega_{\perp}^{\mathrm{Rb}}, \omega_{z}^{\mathrm{Cs}}, \omega_{\perp}^{\mathrm{Cs}}\right)=2 \pi \times(3.89,32.2,4.55,40.2) \mathrm{Hz}$, with longitudinal and transverse shifts of $\sim 1 \mu \mathrm{m}$ in their centers [41,53], and we use scattering lengths $\left(a_{\mathrm{Rb}, \mathrm{Rb}}, a_{\mathrm{Cs}, \mathrm{Cs}}, a_{\mathrm{Rb}, \mathrm{Cs}}\right)=(100,280,650) \mathrm{Bohr}$ radii. Temperature is quenched from $T_{0}=80 \mathrm{nK}$ to $T=20 \mathrm{nK}$. In the first part of this work, we use initial chemical potentials $\mu_{\mathrm{Rb}} / k_{\mathrm{B}}=2.13$ $\mathrm{nK}, \mu_{\mathrm{Cs}}=0.956 \mu_{\mathrm{Rb}}\left(\mu_{\mathrm{Rb}}=1.38 \hbar \omega_{\perp}^{\mathrm{Rb}}, \mu_{\mathrm{Cs}}=1.05 \hbar \omega_{\perp}^{\mathrm{Cs}}\right.$ ), and rates $\gamma_{\mathrm{Rb}}=\gamma_{\mathrm{Cs}}=0.263 \mathrm{~s}^{-1}$ (equivalent to $\hbar \gamma_{k} / k_{\mathrm{B}} T=10^{-4}$ ), with $\mu_{\mathrm{Rb}}^{\prime}=\mu_{\mathrm{Rb}}$ and $\mu_{\mathrm{Cs}}^{\prime}=7.34 \mu_{\mathrm{Cs}}$. The initial condition is an equilibrated state with no single condensate mode $(r \sim 1)$. In (f) inset, we also plot the average defect number $\left\langle N_{\text {sol }}\right\rangle$ immediately following finite-duration quenches with $\left(T_{k}, \mu_{k}\right)$ ramped linearly from initial to final values (as given above) over time $t_{\mathrm{R}}$. Lines qualitatively show potential defect number scalings with $t_{\mathrm{R}}$.

providing strong evidence that the density profiles emerging over experimentally relevant time scales are determined by the history of spontaneous defect formation and subsequent dynamics (Fig. 1). This long-term memory is facilitated by the added stability provided by formation, during coarse-graining dynamics, of a composite dark-bright solitary wave [54-59] defect, a process found to be robust to perturbations, but sensitive to shot-to-shot fluctuations and global parameter changes.

While the equilibrium states of immiscible two-component BECs in the mean-field approximation are known to display various symmetric and asymmetric structures $[53,60]$ similar to those observed in experiment [40,41], we show similar structures emerging as part of a rich nonequilibrium dynamical behavior long before the system reaches an equilibrium state. Our work offers insight into the complexities of two-component BEC formation, and suggests caution when concluding that an experimental immiscible two-component $\mathrm{BEC}$ has reached a true equilibrium state.

\section{QUENCH PROTOCOL AND MODELING DETAILS}

Our quench scheme closely follows that of Ref. [31], namely, a sudden temperature and chemical potential quench, appropriately generalized to two components. We consider a prolate (aspect ratio $\approx 10$ ) two-component atomic cloud of approximately $1.4 \times 10^{6}{ }^{87} \mathrm{Rb}$ and $8 \times 10^{5}{ }^{133} \mathrm{Cs}$ atoms, equilibrated in slightly displaced traps at $T_{0}=80 \mathrm{nK}$ (close to the ideal gas critical temperature $T_{\mathrm{c}}$ ).

Based on a characteristic example (Fig. 2), in which neither component initially possesses a single macroscopically occupied mode, we analyze the role of fluctuations and quench parameters [Figs. 3 and 4(a)], under the assumption that Rb condenses fastest. We then analyze a range of other initial states and quenches, and critically discuss our main assumptions [Figs. 4(b), 5, and 2(f) inset]. Within current computational constraints, this provides a qualitative characterization of the dynamical phase diagram.

Dynamical two-component BEC simulations to date have been based on coupled ordinary $[54,55,61,62]$ or dissipative $[20,63,64]$ Gross-Pitaevskii equations (GPEs), or on classical field [65], truncated Wigner [44,45], or ZNG (coupled GPE-Boltzmann) [66] methods. The effects of thermal fluctuations during condensate growth are best captured by three-dimensional (3D) coupled stochastic projected GrossPitaevskii equations [52,67,68],

$$
d \psi_{k}=\mathcal{P}\left\{-\frac{i}{\hbar} L_{k} \psi_{k}+\frac{\gamma_{k}}{k_{\mathrm{B}} T}\left(\mu_{k}-L_{k}\right) \psi_{k}\right\} d t+d W_{k},
$$

describing the evolution of highly occupied "classical" modes $\psi_{k}$, where $k$ labels the species (here Rb or Cs). Here $L_{k}=$ $-\hbar^{2} \nabla^{2} / 2 m_{k}+V_{k}+4 \pi \hbar^{2}\left(a_{k k}\left|\psi_{k}\right|^{2} / m_{k}+a_{k j}\left|\psi_{j}\right|^{2} / M_{k j}\right)$, for scattering lengths $a_{k k}$ (intraspecies) and $a_{k j}$ (interspecies), atomic masses $m_{k}$, and reduced mass $M_{k j}$. The "c-field" region defined by projector $\mathcal{P}$ consists of single-particle modes $\phi_{l}$ with energies below a carefully selected energy cutoff [69], which is coupled to the above-cutoff reservoir at temperature $T$ via growth (described by the species-dependent rates $\gamma_{k}$ ) and noise (described by $d W_{k}$ [70]) processes. To simulate two-component condensate formation, we first obtain an initial state by numerically propagating Eq. (1) to 
equilibrium for initial chemical potentials $\mu_{k}$ and common temperature $T_{0} \gtrsim T_{\mathrm{C}}$, and then instantaneously quench these parameters to final values $T<T_{\mathrm{C}}$ and $\mu_{k}^{\prime} \geqslant \mu_{k}$. We extract the time-dependent condensate mode using the Penrose-Onsager (PO) criterion [71], by seeking a macroscopically occupied eigenmode of the one-body density matrix in short-time averages over a single trajectory [67].

\section{COUPLED DYNAMICS SIMULATIONS}

\section{A. Typical coupled dynamics simulation}

A typical numerical run of the post-instantaneous-quench dynamics demonstrating the formation of a composite defect for a given initial state (see figure caption for parameters) is shown in Fig. 2, a result with which subsequent results will be compared. Snapshots (left panels) of the c-field density (a), condensate mode density (b), and condensate phase (c) reveal three characteristic dynamical stages: (i) a short postquench "condensation onset" stage with strongly nonequilibrium dynamics (for $t \lesssim 0.1 \mathrm{~s}$ ) in which multiple quench-induced defects proliferate; (ii) a rapid relaxation stage (here $t \lesssim 0.5 \mathrm{~s}$, featuring rapid $\mathrm{Rb}$ growth), dominated by defect coarse-graining dynamics, leading to a metastable equilibrium state with a number of long-lived defects [this number can be zero, one (as shown here), or potentially more]; (iii) a slow evolution towards global equilibrium, during which the other component (here Cs) may (but need not) also condense. While the details of the coupled dynamics are sensitive to global system and quench parameters, our subsequent analysis confirms that the occurrence of these three stages is generic. Within our model, for a given set of quench parameters, the long-term evolution tends to favor the dominance of either $\mathrm{Rb}$ or $\mathrm{Cs}$.

The right panels in Fig. 2 show the dynamics of the PO condensate atom number $N_{0}(\mathrm{~d})$, the occupation ratio, $r$ of the condensate mode to the next-most-highly occupied mode (e), and the number of soliton defects (f). While initially $r \sim 1$, it increases rapidly postquench as a macroscopically occupied condensate mode emerges. Coherence growth, caused by the suppression of phase fluctuations during condensation $[15,16]$, coincides with the rapid decrease in the defect numbers due to merging and decay [33]. Due to the larger initial $\mathrm{Rb}$ atom number, $\mathrm{Cs}$ condensation follows that of $\mathrm{Rb}$ [40]; moreover, because our simulations do not include evaporative cooling, we find that Cs condensation is associated with a gradual decrease in the $\mathrm{Rb}$ condensate.

Depending on dimensionality [29], the emerging defect could also be a vortex or solitonic vortex [72], whose infilling would create a (solitonic) vortex-bright soliton [73] rather than a dark-bright soliton. However, our geometry and parameters, which have $\left\{\mu_{\mathrm{Rb}}, \mu_{\mathrm{Rb}}^{\prime}\right\}<4 \hbar \omega_{\perp}^{\mathrm{Rb}}$ and $\left\{\mu_{\mathrm{Cs}}, \mu_{\mathrm{Cs}}^{\prime}\right\}<8 \hbar \omega_{\perp}^{\mathrm{Cs}}$, favor 1D solitonic defects [72]. In Fig. 2, the initial multiple defects in the fastest condensing component eventually lead to exactly one long-lived defect, which acts as a tight mobile microtrap [11], facilitating rapid condensation of the other component. Growth of the bright (infilling) component in the microtrap (which stabilizes the dark soliton against decay [63]) leads to a spontaneously generated dark-bright soliton; for this particular simulation, this soliton forms close to the trap center.
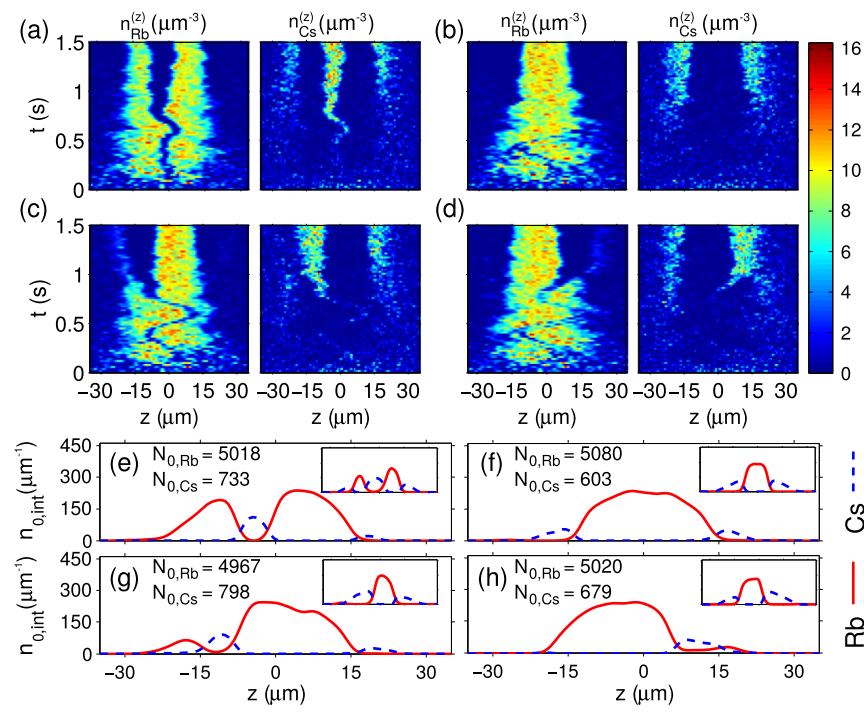

FIG. 3. Evolution of four different postquench random noise sequences, with identical prequench states. (a)-(d) show the c-field density evolution [shown here via $n_{k}^{(z)}=n_{k}(x=0, y=0, z)$ ], and (e)-(h) the condensate density profiles after $1 \mathrm{~s}$ (insets $2 \mathrm{~s}$; ticks on inset axes correspond to those on the main figure axes). After this time the $\mathrm{Rb}$ defect has either been stabilized by Cs infilling (e); fully decayed within $\mathrm{Rb}(\mathrm{f})$; or decayed to the left ( $\mathrm{g}$ ) or right (h) edge of the condensate. Quench parameters are as in Fig. 2.

Over longer time scales, the growing mean-field potential converts this dark-bright solitary wave into a fixed domain wall $[74,75]$. A Cs condensate also subsequently forms at the system edges, slightly compressing the Rb condensate towards the trap center. This interplay between condensate growth, defect formation, and dissipative evolution in the first component, and condensate growth in the second componentalongside increasing mean-field repulsion between the coforming immiscible condensates-leads to interesting features that should be observable in current experiments.

In the following we consider (i) the role of shot-to-shot fluctuations (Fig. 3); (ii) dependence on growth rates and final quench parameters when starting from quasicondensate initial states (Fig. 4); (iii) changes to the evolution due to less coherent initial states (Fig. 5), also demonstrating that the scenario of a defect in $\mathrm{Rb}$ leading to $\mathrm{Cs}$ infilling can be effectively reversed for particular parameters and time scales [Fig. 5(f), $t=0.25 \mathrm{~s}$ ]; (iv) finite-duration quenches, showing that our qualitative findings also hold for quenches of realistic experimental duration [Fig. 2(f), inset].

\section{B. Shot-to-shot variations}

We investigate sensitivity to fluctuations by looking at different numerical runs (loosely corresponding to different experimental runs) for the same fixed quench sequence [Figs. 3(a)-3(d)]. Such runs feature statistically identical condensate number evolution [see error bars in Fig. 4(a)]. Figure 3(a) reveals a three-stage evolution in the "reference" case of Fig. 2: slow-moving solitonic defect, near-stationary dark-bright solitary wave, and eventual static domain wall structure. Figures 3(b)-3(d) reveal alternative (and roughly 
equally likely) outcomes for different (same mean amplitude) postquench dynamical noise realizations, describing thermal fluctuations. Figure 3(c) shows slower Cs growth, in an initially shallower (hence more rapidly moving) dark solitary-wave microtrap. Unlike Fig. 3(a), where dark solitary wave decay and stabilization occur at the trap center, Figs. 3(c)-3(d) show this process occurring either to the left (c), or to the right (d), illustrating the crucial role of the motion of the decaying dark solitary wave [76]. Figure 3(b) illustrates the potential for coexistence of more than one (shallow) defect for an appreciable time [see also Fig. 4(d) for $t \lesssim 0.5 \mathrm{~s}$ ], which typically decay without $\mathrm{Cs}$ growth in the solitonic microtraps. In this case, $\mathrm{Cs}$ only condenses around $\mathrm{Rb}$, minimizing meanfield repulsion. Figures 3(e)-3(h) show the condensate profile after $1 \mathrm{~s}$ (insets $2 \mathrm{~s}$ ), consisting of either a large Cs structure in the middle separating the $\mathrm{Rb}$ [inset to Fig. 3(e)], or a large $\mathrm{Rb}$ structure enclosed by Cs [insets to Figs. 3(f)-3(h)]. Further evolution of case (a) (to $\gtrsim 3$ s) also yields an $\mathrm{Rb}$ structure enclosed by $\mathrm{Cs}$, after gradual merging of the two Rb peaks.

We have confirmed that the qualitative findings above are unchanged when using different (same temperature) thermalized initial states in the same simulations, loosely corresponding to different experimental runs. In particular, although different, randomly generated, initial (prequench) states yield different postquench defect formation dynamics, we find that at least three out of nine simulations with different initial and dynamical noise reveal clear evidence of spontaneous dark-bright soliton formation, with two of the emerging composite defects being relatively deep and central. Despite the differences in emergent density profile, condensate atom number evolution is the same [within statistical variations, see error bars in Fig. 4(a)], irrespective of the formation, stabilization, or decay of the dark-bright solitary wave. The importance of dark-bright solitary waves in the early stages of formation is further confirmed through dissipative GPE simulations, showing that even perfectly imprinted multiple dark-bright solitons quickly coalesce into a single long-lived dark-bright soliton, with rapid dynamical mean-field stabilization. Importantly, we find that the presence, or absence, of small asymmetries $(\sim 1 \mu \mathrm{m})$ in the trap minima alter only the details, but not the qualitative structures, of the emerging density profiles, indicating that shot-to-shot and thermal fluctuations can strongly suppress experimentally relevant trap imperfections (whose role can dominate meanfield simulations [53]).

\section{Dependence on global quench parameters}

Distinct dynamical regimes occurring for different quench parameters are shown in Fig. 4. We focus on the dependence of $N_{0}$ on variations of the chemical potentials $\mu_{k}^{\prime}$ and growth rates $\gamma_{k}$ for fixed initial quasicondensate states. With $\gamma_{\mathrm{Rb}}=\gamma_{\mathrm{Cs}}$ fixed, we find $\mathrm{Rb}$ dominates the evolution for a broad range of $\mu_{\mathrm{Rb}}^{\prime}>\mu_{\mathrm{Rb}}$ [squares and black lines in Fig. 4(a)]. Here, spontaneously generated defects tend to decay within $\mathrm{Rb}$, with Cs not condensing into a dark-bright solitary wave microtrap but instead at the trap edges [Fig. 4(c)]. For quenches with large $\mu_{\text {Cs }}^{\prime}$ [Fig. 4(a), green triangles], despite the early atom number evolution remaining practically unchanged, Cs condensation rapidly overtakes that of $\mathrm{Rb}$, which gradually disappears
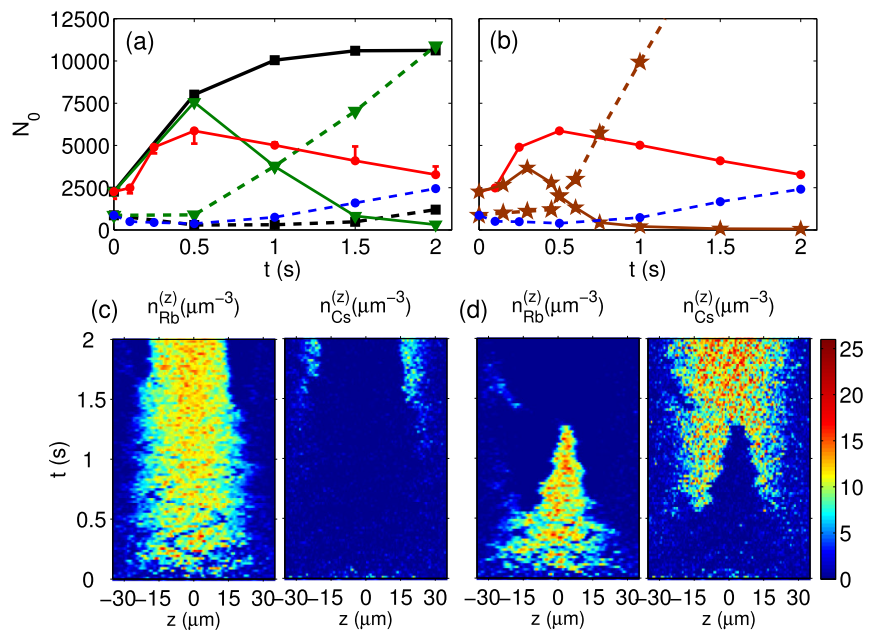

FIG. 4. Dependence of evolution on quench parameters. (a) shows condensate number evolution ( $\mathrm{Rb}$ solid, $\mathrm{Cs}$ dashed) for different final chemical potentials $\mu_{k}^{\prime}$; standard parameters $\mu_{\mathrm{Rb}}^{\prime}=$ $\mu_{\mathrm{Rb}}, \mu_{\mathrm{Cs}}^{\prime}=7.34 \mu_{\mathrm{Cs}}$ (red and blue circles); $\mu_{\mathrm{Rb}}^{\prime}=2.63 \mu_{\mathrm{Rb}}, \mu_{\mathrm{Cs}}^{\prime}=$ $7.34 \mu_{\mathrm{Cs}}$ [black squares, example of c-field density evolution shown in (c)]; $\mu_{\mathrm{Rb}}^{\prime}=\mu_{\mathrm{Rb}}, \mu_{\mathrm{Cs}}^{\prime}=11.9 \mu_{\mathrm{Cs}}$ [green triangles, example of c-field density evolution shown in (d)]. Error bars in (a) for standard parameters indicate the standard deviation over six numerical trajectories. (b) shows condensate number evolution for different rates $\gamma_{k}$; standard parameters $\gamma_{\mathrm{Cs}}=\gamma_{\mathrm{Rb}}=0.263 \mathrm{~s}^{-1}$ (red and blue circles), and $\gamma_{\mathrm{Cs}}=10 \gamma_{\mathrm{Rb}}=2.63 \mathrm{~s}^{-1}$ (brown stars).

[Fig. 4(d)]. We have also investigated the effect of the growth rate on the condensate evolution (standard estimates [67] suggest that $\gamma_{\mathrm{Cs}} \sim 10 \gamma_{\mathrm{Rb}}$ ), and find that increasing $\gamma_{\mathrm{Cs}} / \gamma_{\mathrm{Rb}}$, for fixed chemical potentials, favors the rapid growth of $\mathrm{Cs}$ (after a brief initial transient of rapid Rb growth) [Fig. 4(b)].

\section{Role of quasicondensation in prequench states}

Our simulations so far exhibit a consistent predominance of rapid $\mathrm{Rb}$ growth initially, set by the prequench conditions, with long-term evolution dictated by the quench parameters. Although our initial states featured no single macroscopically occupied state in either component, both initial states exhibited quasicondensation, with multiple states having occupations greater than half that of the most occupied state $[\approx 10$ states for $\mathrm{Rb}$ (largest occupation $\approx 2200$ ), and $\approx 5$ states for Cs (largest occupation $\approx 1100$ )]. To demonstrate the generality of our findings, Fig. 5 shows the effects of decreasing the initial $\mathrm{Rb}$ quasicondensation, i.e., changing $\mu_{\mathrm{Rb}}$ from $>0$ [Fig. 5(c)] to $\approx 0$ [Fig. 5(d)] and $<0$ [Fig. 5(e)], while allowing for an initial, more pronounced, Cs quasicondensate (through the increased $\mu_{\mathrm{Cs}}=2.1 \hbar \omega_{\perp}^{\mathrm{Cs}}$ ). This leads to a large Cs condensate forming before significant $\mathrm{Rb}$ condensate growth. Under these conditions we find that enhancing the final $\mathrm{Rb}$ chemical potential, $\mu_{\mathrm{Rb}}^{\prime}$ [Fig. 5(f)], can lead either to approximately equal $\mathrm{Rb}$ and $\mathrm{Cs}$ condensate number, or to the generation of a quasistable Cs defect enabling the short-time formation and entrapment of an $\mathrm{Rb}$ condensate (at $t=0.25 \mathrm{~s}$ ).

\section{E. Effects of finite quench duration}

As a further check on the generality of our results, we also consider finite-duration quenches in which $\left(T_{k}, \mu_{k}\right)$ are 

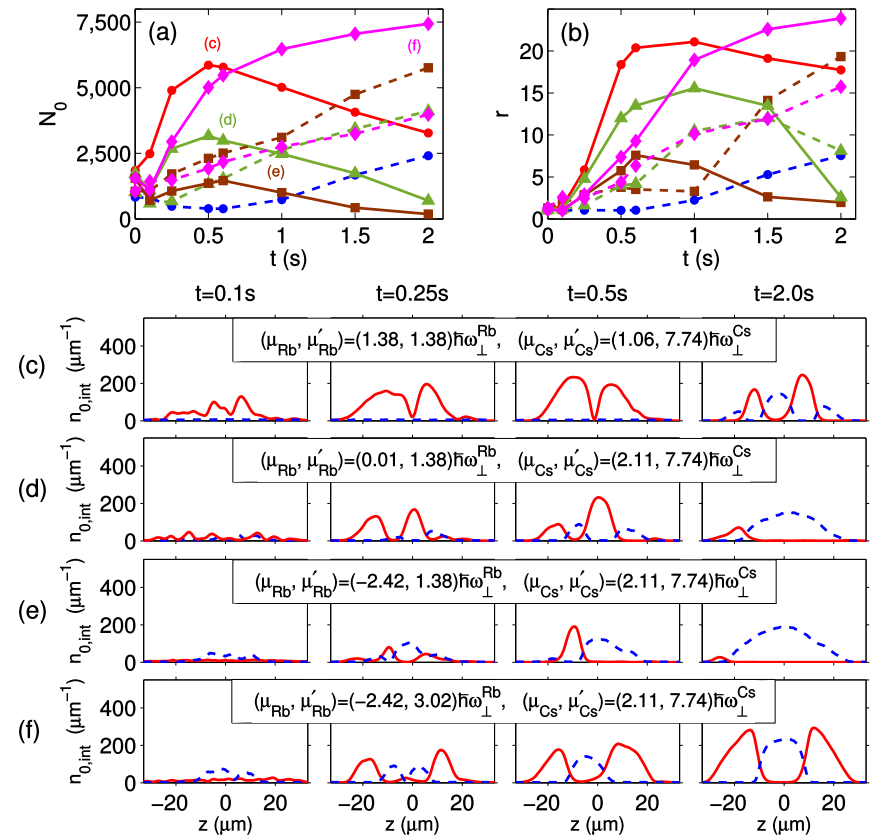

FIG. 5. Evolution of (a) condensate atom number, (b) occupation ratio $r$, and (c)-(f) coupled $1 \mathrm{D}$ integrated condensate density profiles for initial states with different amount of coherence in the $\mathrm{Rb}$ initial state. Shown are cases with $\mu_{\mathrm{Cs}}>0$ and (c) $\mu_{\mathrm{Rb}}=$ $1.38 \hbar \omega_{\perp}^{\mathrm{Rb}}$ (corresponding to "reference" Fig. 2 data, plotted only for ease of comparison here), (d) $\mu_{\mathrm{Rb}}=0.01 \hbar \omega_{\perp}^{\mathrm{Rb}}$, and (e)-(f) $\mu_{\mathrm{Rb}}=$ $-2.42 \hbar \omega_{\perp}^{\mathrm{Rb}}$; (f) differs from (e) only in the larger postquench $\mathrm{Rb}$ chemical potential $\left[\mu_{\mathrm{Rb}}^{\prime}=3.02 \hbar \omega_{\perp}^{\mathrm{Rb}}\right.$ in (f), as opposed to $1.38 \hbar \omega_{\perp}^{\mathrm{Rb}}$ in (e)]. Note that for (d)-(f) $\mu_{\mathrm{Cs}}$ is increased to $2.11 \hbar \omega_{\perp}^{\mathrm{Cs}}$.

ramped linearly from the same initial to final values as in Fig. 2 over time $t_{\mathrm{R}}$. For short but realistic ramp durations $\left(t_{\mathrm{R}} \lesssim \omega_{z}^{-1}\right)$ we find identical short-term $\left(t \lesssim t_{\mathrm{R}}\right)$ and very similar long-term $(t \sim 500-1000 \mathrm{~ms})$ dynamics, supporting our findings about the emergence of a single relatively deep dark soliton $(\mathrm{Rb})$, partly infilled by the other component $(\mathrm{Cs})]$; this is in qualitative agreement with earlier findings for singlecomponent systems (see Supplementary Information in [31]). The scaling of the immediate postquench defect number for finite-duration quenches with $t_{\mathrm{R}}$, starting from a state exhibiting quasicondensation ( $T_{0} \gtrsim T_{\mathrm{c}}$ ), is shown in Fig. 2(f), inset (for the relevant time scales shorter than the characteristic time scale for the emergence of a composite defect).

As the physical scheme considered here does not fully conform to the standard adiabatic-impulse-adiabatic scenario of the Kibble-Zurek mechanism [29], we cautiously avoid directly linking our findings for the study of the soliton number to Kibble-Zurek exponents. Nonetheless and rather remarkably we find some qualitative resemblance to scalings observed in the short- and long-term evolution of spinor gases quenched from miscible to immiscible in homogeneous or harmonically trapped systems [44-46,49,77].

\section{EXPERIMENTAL RELEVANCE}

Recent experiments have explored the emergent immiscible density profiles following the gradual sympathetic cooling of
${ }^{87} \mathrm{Rb}-{ }^{85} \mathrm{Rb}$ [40] or ${ }^{87} \mathrm{Rb}-{ }^{133} \mathrm{Cs}$ [41] mixtures. Although our simulations feature sudden quenches, we can still draw some interesting, albeit indirect, analogies to these experiments: (i) Both experiments sometimes found (depending on parameters) a condensate in only one component, in qualitative agreement with the long-term evolution of our stochastic simulations. (ii) Our simulations reveal long-lived metastable phase-separated profiles resembling those seen in experiment [41]. While the energy of the immiscible system is generally lowered by reducing the number of domains, larger aspect ratios add an energy barrier to the process of reduction; the experiment of Ref. [40] (with larger aspect ratio) reported metastable states with lifetimes exceeding 1 $\mathrm{s}$, consistent with our simulation time scales. (iii) The relative time scales for condensation in the two components influence the form of the emergent profiles, as the component condensing second (typically with fewer initial atoms, and thus lower critical temperature) can only do so when the density of the other component is low [40,41]; this explains why, over the probed time scale, simulations in the scenario of Fig. 3 most commonly exhibit a central $\mathrm{Rb}$ condensate surrounded by the more massive Cs (a feature not easily reproduced at equilibrium [53]). The long-term evolution will, in principle, erase fluctuations from the formation dynamics (although in practice this time scale may be too long to be experimentally relevant). Indeed, longer term evolution in the scenario of Fig. $2(t \gtrsim 3 \mathrm{~s})$ exhibits coalescence of the two Rb structures into a single structure surrounded by Cs. This coalescence often occurs shortly after the $\mathrm{Rb}$ condensate number decreases below that of Cs, providing a plausible indirect explanation for the observation of such a regime in Ref. [41].

\section{CONCLUSIONS}

We have qualitatively analyzed the formation dynamics of immiscible two-component condensates following a sudden temperature quench, elucidating the importance of composite defect formation and dynamics. While our model is computationally challenging (even the coupled dissipative model, without noise, has eight independent parameters), analysis of over 100 three-dimensional stochastic simulations has enabled us to broadly classify the dynamics into three evolution stages: (i) condensation of the fastest growing component, with dynamics mainly determined by the prequench state and final temperature, in which multiple defects are spontaneously formed; (ii) fluctuation-determined coarse-graining dynamics, during which defects in the fastest growing component coalesce and gradually decay, potentially yielding a small number of longlived defects; and (iii) relaxation, with long-term dynamics set by the quench parameters, during which the second component condenses in regions of low mean-field density. Importantly, a statistically non-negligible fraction ( $\gtrsim 30 \%)$ of simulations exhibit a composite defect, whose spontaneous formation and subsequent evolution lead to stark shot-to-shot variations in the observable phase-separated density profiles over a broad range of experimentally relevant time scales. We have verified the generic nature of our observations both by confirming that our findings also hold for realistic finite-duration quenches, and 
that for suitable parameter choices, a defect-induced microtrap can emerge in either species.

While the prevailing composite defect in our inhomogeneous system is a dark-bright solitary wave, higher dimensional analogs are also possible, and the related "core condensation" dynamics in the context of condensed matter and high-energy physics remain only partly understood even in uniform systems [28,30]. Controlled experiments with cold atoms could help shed further light on this important problem by, e.g., statistically analyzing the evolution of density profiles following quenched evaporative cooling of binary gases initially near the critical temperature.
Data supporting this publication is openly available under an Open Data Commons Open Database License [79].

\section{ACKNOWLEDGMENTS}

We acknowledge funding from the U.K. EPSRC (Grant Nos. EP/K03250X/1 and EP/K030558/1). I.K.L. and S.C.G. were supported by the Ministry of Science and Technology, Taiwan (Grant No. 103-2112-M-018-002-MY3). T.P.B. was supported by the John Templeton Foundation via the Durham Emergence Project (http://www.dur.ac.uk/emergence).
[1] M. C. Cross and P. C. Hohenberg, Rev. Mod. Phys. 65, 851 (1993).

[2] E. Meron, Phys. Rep. 218, 1 (1992).

[3] J. D. Murray, Mathematical Biology. II, Spatial Models and Biomedical Applications, Interdisciplinary Applied Mathematics (Springer, New York, 2003).

[4] H. T. C. Stoof, Phys. Rev. Lett. 78, 768 (1997).

[5] Y. Kagan and B. V. Svistunov, Phys. Rev. Lett. 79, 3331 (1997).

[6] D. V. Semikoz and I. I. Tkachev, Phys. Rev. D 55, 489 (1997).

[7] E. Levich and V. Yakhot, J. Phys. A 11, 2237 (1978).

[8] H.-J. Miesner, D. M. Stamper-Kurn, M. R. Andrews, D. S. Durfee, S. Inouye, and W. Ketterle, Science 279, 1005 (1998).

[9] C. W. Gardiner, M. D. Lee, R. J. Ballagh, M. J. Davis, and P. Zoller, Phys. Rev. Lett. 81, 5266 (1998).

[10] M. J. Bijlsma, E. Zaremba, and H. T. C. Stoof, Phys. Rev. A 62, 063609 (2000)

[11] D. M. Stamper-Kurn, H.-J. Miesner, A. P. Chikkatur, S. Inouye, J. Stenger, and W. Ketterle, Phys. Rev. Lett. 81, 2194 (1998).

[12] H. T. C. Stoof and M. J. Bijlsma, J. Low Temp. Phys. 124, 431 (2001).

[13] M. Köhl, M. J. Davis, C. W. Gardiner, T. W. Hänsch, and T. Esslinger, Phys. Rev. Lett. 88, 080402 (2002).

[14] N. P. Proukakis, J. Schmiedmayer, and H. T. C. Stoof, Phys. Rev. A 73, 053603 (2006).

[15] M. Hugbart, J. A. Retter, A. F. Varón, P. Bouyer, A. Aspect, and M. J. Davis, Phys. Rev. A 75, 011602 (2007).

[16] S. Ritter, A. Öttl, T. Donner, T. Bourdel, M. Köhl, and T. Esslinger, Phys. Rev. Lett. 98, 090402 (2007).

[17] T. Donner, S. Ritter, T. Bourdel, A. Öttl, M. Köhl, and T. Esslinger, Science 315, 1556 (2007).

[18] M. C. Garrett, A. Ratnapala, E. D. van Ooijen, C. J. Vale, K. Weegink, S. K. Schnelle, O. Vainio, N. R. Heckenberg, H. Rubinsztein-Dunlop, and M. J. Davis, Phys. Rev. A 83, 013630 (2011).

[19] N. Navon, A. L. Gaunt, R. P. Smith, and Z. Hadzibabic, Science 347, 167 (2015).

[20] S. Ronen, J. L. Bohn, L. E. Halmo, and M. Edwards, Phys. Rev. A 78, 053613 (2008).

[21] E. del Valle, D. Sanvitto, A. Amo, F. P. Laussy, R. André, C. Tejedor, and L. Viña, Phys. Rev. Lett. 103, 096404 (2009).

[22] G. Nardin, K. G. Lagoudakis, M. Wouters, M. Richard, A. Baas, R. André, L. S. Dang, B. Pietka, and B. Deveaud-Plédran, Phys. Rev. Lett. 103, 256402 (2009).
[23] A.-W. de Leeuw, H. T. C. Stoof, and R. A. Duine, Phys. Rev. A 88, 033829 (2013).

[24] A. Chiocchetta and I. Carusotto, Phys. Rev. A 90, 023633 (2014).

[25] T. W. B. Kibble, J. Phys. A 9, 1387 (1976).

[26] W. H. Zurek, Nature 317, 505 (1985).

[27] W. Zurek, Phys. Rep. 276, 177 (1996).

[28] T. Kibble and A. Srivastava, J. Phys. Condens. Matter 25, 400301 (2013).

[29] A. del Campo, T. W. B. Kibble, and W. H. Zurek, J. Phys. Condens. Matter 25, 404210 (2013).

[30] N. D. Antunes, P. Gandra, R. J. Rivers, and A. Swarup, Phys. Rev. D 73, 085012 (2006).

[31] C. N. Weiler, T. W. Neely, D. R. Scherer, A. S. Bradley, M. J. Davis, and B. P. Anderson, Nature 455, 948 (2008).

[32] G. Lamporesi, S. Donadello, S. Serafini, F. Dalfovo, and G. Ferrari, Nat. Phys. 9, 656 (2013).

[33] S. Donadello, S. Serafini, M. Tylutki, L. P. Pitaevskii, F. Dalfovo, G. Lamporesi, and G. Ferrari, Phys. Rev. Lett. 113, 065302 (2014).

[34] L. Corman, L. Chomaz, T. Bienaimé, R. Desbuquois, C. Weitenberg, S. Nascimbène, J. Dalibard, and J. Beugnon, Phys. Rev. Lett. 113, 135302 (2014).

[35] W. H. Zurek, Phys. Rev. Lett. 102, 105702 (2009).

[36] B. Damski and W. H. Zurek, Phys. Rev. Lett. 104, 160404 (2010).

[37] E. Witkowska, P. Deuar, M. Gajda, and K. Rzążewski, Phys. Rev. Lett. 106, 135301 (2011).

[38] A. del Campo, A. Retzker, and M. B. Plenio, New J. Phys. 13, 083022 (2011).

[39] G. Modugno, M. Modugno, F. Riboli, G. Roati, and M. Inguscio, Phys. Rev. Lett. 89, 190404 (2002).

[40] S. B. Papp, J. M. Pino, and C. E. Wieman, Phys. Rev. Lett. 101, 040402 (2008).

[41] D. J. McCarron, H. W. Cho, D. L. Jenkin, M. P. Köppinger, and S. L. Cornish, Phys. Rev. A 84, 011603 (2011).

[42] A. Lercher, T. Takekoshi, M. Debatin, B. Schuster, R. Rameshan, F. Ferlaino, R. Grimm, and H.-C. Nägerl, Eur. Phys. J. D 65, 3 (2011).

[43] S.-W. Su, S.-C. Gou, A. S. Bradley, O. Fialko, and J. Brand, Phys. Rev. Lett. 110, 215302 (2013).

[44] J. Sabbatini, W. H. Zurek, and M. J. Davis, Phys. Rev. Lett. 107, 230402 (2011).

[45] J. Sabbatini, W. H. Zurek, and M. J. Davis, New J. Phys. 14, 095030 (2012). 
[46] J. Hofmann, S. S. Natu, and S. Das Sarma, Phys. Rev. Lett. 113, 095702 (2014).

[47] L. E. Sadler, J. M. Higbie, S. R. Leslie, M. Vengalattore, and D. M. Stamper-Kurn, Nature 443, 312 (2006).

[48] H. Saito, Y. Kawaguchi, and M. Ueda, J. Phys. Condens. Matter 25, 404212 (2013).

[49] T. Świsłocki, E. Witkowska, J. Dziarmaga, and M. Matuszewski, Phys. Rev. Lett. 110, 045303 (2013).

[50] E. Witkowska, J. Dziarmaga, T. Świsłocki, and M. Matuszewski, Phys. Rev. B 88, 054508 (2013).

[51] D. M. Stamper-Kurn and M. Ueda, Rev. Mod. Phys. 85, 1191 (2013).

[52] S. De, D. L. Campbell, R. M. Price, A. Putra, B. M. Anderson, and I. B. Spielman, Phys. Rev. A 89, 033631 (2014).

[53] R. W. Pattinson, T. P. Billam, S. A. Gardiner, D. J. McCarron, H. W. Cho, S. L. Cornish, N. G. Parker, and N. P. Proukakis, Phys. Rev. A 87, 013625 (2013).

[54] T. Busch and J. R. Anglin, Phys. Rev. Lett. 87, 010401 (2001).

[55] P. Öhberg and L. Santos, Phys. Rev. Lett. 86, 2918 (2001).

[56] U. Shrestha, J. Javanainen, and J. Ruostekoski, Phys. Rev. Lett. 103, 190401 (2009).

[57] C. Becker, S. Stellmer, P. Soltan-Panahi, S. Dörscher, M. Baumert, E.-M. Richter, J. Kronjäger, K. Bongs, and K. Sengstock, Nat. Phys. 4, 496 (2008).

[58] C. Hamner, J. J. Chang, P. Engels, and M. A. Hoefer, Phys. Rev. Lett. 106, 065302 (2011).

[59] C. Hamner, Y. Zhang, J. J. Chang, C. Zhang, and P. Engels, Phys. Rev. Lett. 111, 264101 (2013).

[60] M. Trippenbach, K. Goral, K. Rzazewski, B. Malomed, and Y. B. Band, J. Phys. B 33, 4017 (2000).

[61] K. Kasamatsu and M. Tsubota, Phys. Rev. A 74, 013617 (2006).

[62] K. Sasaki, N. Suzuki, and H. Saito, Phys. Rev. A 83, 033602 (2011).

[63] V. Achilleos, D. Yan, P. G. Kevrekidis, and D. J. Frantzeskakis, New J. Phys. 14, 055006 (2012).
[64] R. W. Pattinson, N. G. Parker, and N. P. Proukakis, J. Phys. Conf. Ser. 497, 012029 (2014).

[65] N. G. Berloff, Phys. Rev. Lett. 94, 120401 (2005).

[66] M. J. Edmonds, K. L. Lee, and N. P. Proukakis, Phys. Rev. A 91, 011602 (2015).

[67] P. B. Blakie, A. S. Bradley, M. J. Davis, R. J. Ballagh, and C. W. Gardiner, Adv. Phys. 57, 363 (2008).

[68] A. S. Bradley and P. B. Blakie, Phys. Rev. A 90, 023631 (2014)

[69] To make our simulations numerically tractable, we use a plane-wave basis without strict de-aliasing (a procedure subtly different from Refs. [67,78]). However, we have confirmed that our main results remain qualitatively similar when the cutoff is changed by $\sim 10 \%$.

[70] Here $d W_{k}$ is complex white noise defined by $\left\langle d W_{k}^{*}(\mathbf{x}, t) d W_{j}\right.$ $\left.\left(\mathbf{x}^{\prime}, t\right)\right\rangle=2 \gamma_{k} \delta_{k j} \delta_{C ; k}\left(\mathbf{x}-\mathbf{x}^{\prime}\right) d t$, where $\delta_{C ; k}\left(\mathbf{x}-\mathbf{x}^{\prime}\right)=\sum_{l}^{E_{l} \leqslant E_{c ; k}}$ $\phi_{l}^{*}\left(\mathbf{x}^{\prime}\right) \phi_{l}(\mathbf{x})$.

[71] O. Penrose and L. Onsager, Phys. Rev. 104, 576 (1956).

[72] J. Brand and W. P. Reinhardt, Phys. Rev. A 65, 043612 (2002).

[73] K. J. H. Law, P. G. Kevrekidis, and L. S. Tuckerman, Phys. Rev. Lett. 105, 160405 (2010).

[74] S. Coen and M. Haelterman, Phys. Rev. Lett. 87, 140401 (2001).

[75] D. M. Stamper-Kurn, H.-J. Miesner, A. P. Chikkatur, S. Inouye, J. Stenger, and W. Ketterle, Phys. Rev. Lett. 83, 661 (1999).

[76] S. P. Cockburn, H. E. Nistazakis, T. P. Horikis, P. G. Kevrekidis, N. P. Proukakis, and D. J. Frantzeskakis, Phys. Rev. Lett. 104, 174101 (2010).

[77] T. Świsłocki, E. Witkowska, J. Dziarmaga, and M. Matuszewski (private communication).

[78] P. B. Blakie, Phys. Rev. E 78, 026704 (2008).

[79] Additional metadata are available at: http://dx.doi.org/10.17634/ 122626-2. Please contact Newcastle Research Data Service at rdm@ncl.ac.uk for access instructions. 